\title{
Late-Onset Lipid Storage Myopathy with Fatal Hepatosteatosis
}

\author{
Arda Yavuz $^{1}$,_Gökçen Ünverengil ${ }^{2}$, Ayşe Nur Toksöz Yıldırım³ ${ }^{3}$, Hatice Şeyma Maraşlı4 IIlyas Tuncer $^{1}$ \\ ${ }^{1}$ Department of Gastroenterology, Istanbul Medeniyet University, School of Medicine, Istanbul, Turkey \\ ${ }^{2}$ Department of Pathology, Istanbul Faculty of Medicine, Istanbul University, Istanbul, Turkey \\ ${ }^{3}$ Department of Pathology, Istanbul Medeniyet University, School of Medicine, Istanbul, Turkey \\ ${ }^{4}$ Department of Internal Medicine, Istanbul Medeniyet University, School of Medicine, Istanbul, Turkey
}

Received: 30/08/2020

Accepted: 01/09/2020

Published: 07/10/2020

How to cite this article: Yavuz A,_Ünverengil G, Yıldırım ANT, Maraşlı HS, Tuncerl. Late-onset lipid storage myopathy with fatal hepatosteatosis. EJCRIM 2020;7: doi:10.12890/2020_001980.

Conflicts of Interests: The Authors declare that there are no competing interests.

This article is licensed under a Commons Attribution Non-Commercial 4.0 License

\section{ABSTRACT}

Hepatosteatosis, a common condition, is increasing in prevalence. It is typically associated with diet, alcohol consumption and obesity. In some cases, a rare genetic disease may be the underlying defect. Lipid storage myopathy (LSM) is a genetic disease caused by lipid metabolism defects. LSM often affects the muscles, heart and liver. Coenzyme Q, riboflavin or carnitine replacement can be beneficial in some cases. We describe a patient who presented with liver failure and was unresponsive to treatment.

\section{LEARNING POINTS}

- Hepatosteatosis can be associated with genetic disease and not just diet.

- Lipid storage disease should be considered in patients presenting with liver disease with hypoglycaemia, muscle weakness and a family history.

- Lipid storage disease is a rare heterogeneous genetic condition that has no specific treatment and requires further research.

\section{KEYWORDS}

Hepatosteatosis, late-onset lipid storage myopathy, lipid storage disease

\section{CASE DESCRIPTION}

In November 2019, a 33-year-old Turkish man was admitted to the Internal Medicine Polyclinic because of weakness, specifically in the neck muscles, and pain. He had no history of systemic disease. His parents were cousins, and his sister and a cousin had both died of liver failure at 15 years of age, possibly of Wilson's disease, as suspected by doctors at the time following abnormal liver function tests. There were no biopsy reports for either patient.

Ceruloplasmin, free copper and 24-hour urine copper were normal in our patient. No Kayser-Fleischer rings were observed, and cranial magnetic resonance imaging was unremarkable. The penicillamine challenge test was administered due to the patient's family history, and results were positive. The patient was admitted for further investigation.

Viral serology and autoimmune panel results were negative. Ferritin level, transferrin saturation, ACE, alpha-1 antitrypsin, creatine kinase (CK) level, and serum protein electrophoresis were all normal. Abdominal ultrasonography (US) showed grade 2 hepatosteatosis and hepatomegaly $(170 \mathrm{~mm})$. The spleen was normal $(110 \mathrm{~mm})$. Portal Doppler US results were unremarkable, and no ascites was observed. 
Cervical MR, performed because of cervical pain, showed cervical lordosis. The tests did not indicate definite disease and results did not meet the criteria for Wilson's disease.

We decided to perform a liver biopsy, which showed macrovesicular hepatosteatosis, which can indicate early stage Wilson's disease (Fig. 1). The biopsy did not show any haemochromatosis or signs of autoimmune hepatitis. Hepatic copper concentration was $47 \mu \mathrm{g} / \mathrm{day}$. Because of the highly suspect family history, we started treatment with d-penicillamine and zinc. Liver enzyme results remained unchanged. However, muscle weakness increased, hypoglycaemia developed, and CK levels rose to $1472 \mathrm{U} / \mathrm{I}$ (Table 1).

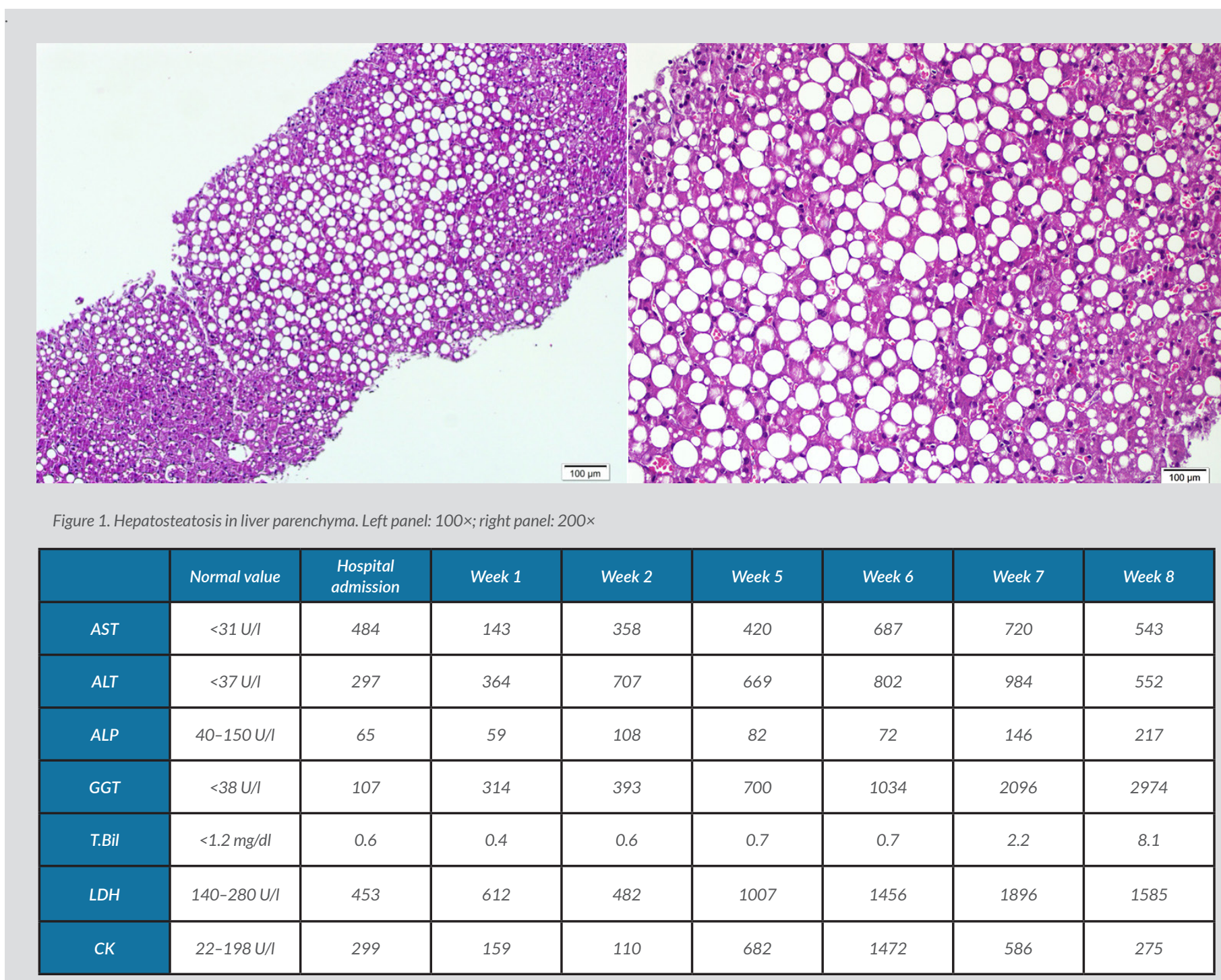

Table 1. The patient's laboratory results

The cousin marriage, hypoglycaemia, muscle weakness and liver failure suggested a differential diagnosis which included glycogen storage disease (GSD), lipid acid oxidation defects, and other genetic disorders. Tests for mucopolisacaridosis and for Gaucher, Fabry and NiemannPick disease were all negative. Echocardiography was normal. Eye examination, joint x-rays and lipid levels were all normal. Serum amino acid levels, urine organic acid levels and tandem MS for inherited metabolic disease were all normal. However the paediatric metabolism department suggested that tandem MS cannot rule out metabolic disease if the disease is not active. They advised us to perform tandem MS, insulin, ACTH, cortisol, ketone, lactate and blood gas analysis if the patient had hypoglycaemia, and indicated that GSD cannot be ruled out with a liver biopsy as an area without storage could have been sampled. They advised genetic analysis if we had a high index of suspicion. We consulted the neurology department regarding the patient. EMG showed general myogenic effects. EEG indicated left asymmetric 
dysfunction in the front of the left hemisphere on regular activity. Neurological examination revealed hypernasality and normal cranial nerves. No bulbar involvement was found. Muscle strength tests showed head flexion $+4 / 5$, upper extremity proximal $+5 / 5$ and distal $+4 / 5$, and lower extremity proximal $+4 / 5$ and distal $+5 / 5$. Acetylcholine receptor antibody tests were negative. The neurology department recommended CK monitoring, a muscle biopsy, vasculitis marker analysis and a search for metabolic myopathies. The muscle biopsy showed massive lipid infiltration (Fig. 2).

A subsequent peripheral smear did not indicate Jordans' anomaly. Carnitine levels were normal. We started coenzyme Q treatment (200 mg per day) and riboflavin (100 mg three times daily) but were unable to initiate any other treatment.

The patient's status worsened, and he did not respond to treatment. He was transferred to the intensive care unit because of respiratory distress and died after 2 days.

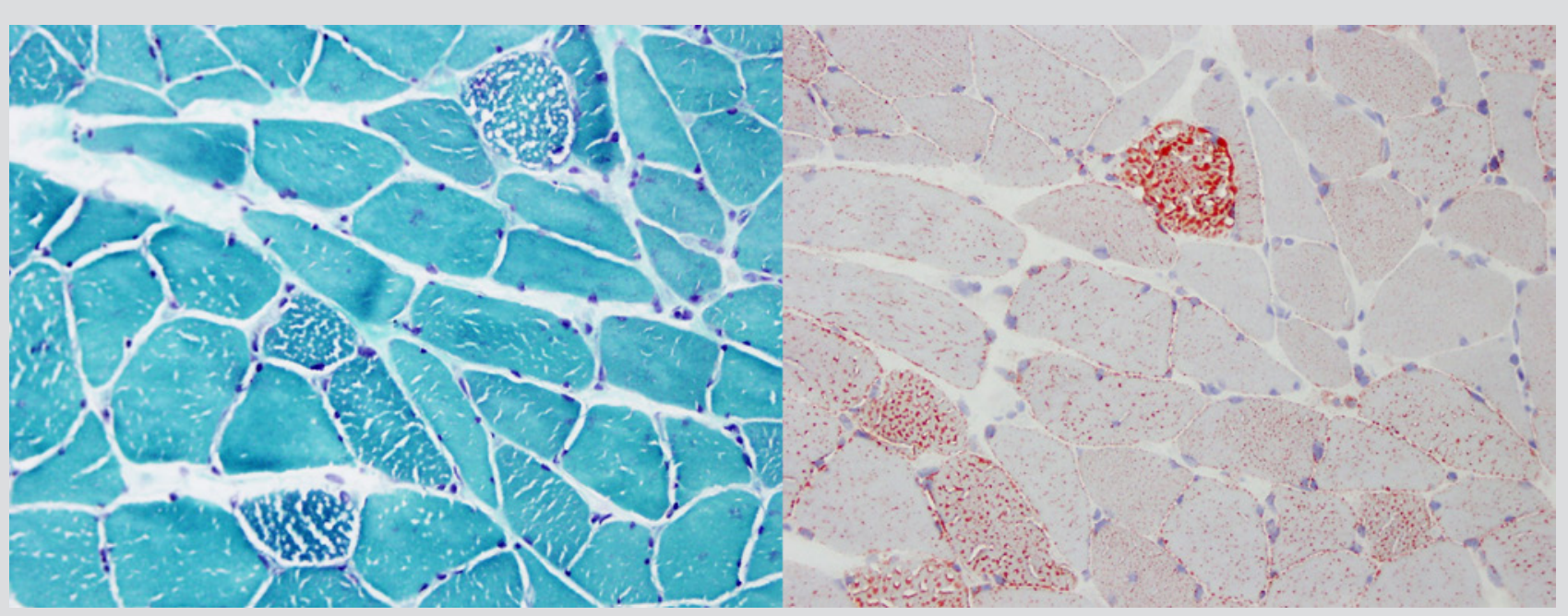

Figure 2. Multiple lipid droplets in type I muscle fibres and smaller lipid droplets in other fibres. Left panel: trichrome stain 200x; right panel: oil red O stain 200x

\section{DISCUSSION}

Late-onset metabolic and genetic liver diseases are difficult to identify because of the low incidence and diagnostic process. The clinical presentation of primary carnitine deficiency consists of hypoketotic hypoglycaemia, hepatomegaly, muscle weakness and congestive heart failure. The diagnosis includes a severe reduction in carnitine levels (free carnitine $<5 \mathrm{mM}$ ). SCL22A5 gene mutation analysis can also help ${ }^{[1]}$. We performed tandem MS, but the carnitine level was normal, but carnitine levels can be normal if the test is not performed at the correct time. Multiple acyl-CoA dehydrogenation deficiency (MADD) is caused by EFTA, EFTB or ETFDH gene mutations, and is riboflavin responsive. This disease is characterized by exercise intolerance, myalgia, progressive proximal muscle weakness, and sometimes hypoglycaemia and metabolic acidosis. There are also case reports which describe hepatic dysfunction, which, however, is not typical ${ }^{[2]}$.

There are two types of neutral lipid storage disease, both of which are characterized by cytoplasmic vacuoles (Jordans' anomalies), which is the most common finding ${ }^{[3]}$. Chanarin-Dorfman syndrome or NLSDI is due to ABHD5 gene mutation, and presents with muscle weakness, ataxia, neurosensory hearing loss, cataracts, nystagmus, strabismus and mental retardation. The other form of NLSD is NLSDM, which is associated with the PNPLA2 gene mutation. This form typically presents with progressive myopathy, often cardiomyopathy, sometimes hepatomegaly, and diabetes mellitus.

Our patient did not have a typical form of these lipid storage myopathy (LSM) subgroups. However, all forms are heterogeneous and probably present as late-onset metabolic disease. Our patient had a disease on the spectrum with hypoglycaemia, hepatosteatosis, dropped head syndrome, muscle weakness, and respiratory muscle weakness. We administered riboflavin and coenzyme Q, but unfortunately could not find the correct treatment.

Finally, this case also shows that hepatosteatosis is not induced just by diet but can be associated with some rare genetic diseases. LSM diseases are a heterogeneous group and can be aggressive and untreatable. Because of their rarity, these study findings are important for future cases. The publication of case reports is essential for investigating the LSM spectrum and increasing awareness. 
European Journal

of Case Reports in

Internal Medicine

\section{REFERENCES}

1. Fu L, Huang M, Chen S. Primary carnitine deficiency and cardiomyopathy. Korean Circ J 2013;43(12):785-792.

2. Behin A, Acquaviva-Bourdain C, Souvannanorath S. Multiple acyl-CoA dehydrogenase deficiency (MADD) as a cause of late-onset treatable metabolic disease. Rev Neurol 2016;172(3):231-241.

3. Tavian D, Colombo R. Improved cytochemical method for detecting Jordans' bodies in neutral lipid storage disease. J Clin Pathol 2007;60:956-958. 